Published in final edited form as:

Clin Rheumatol. 2017 January ; 36(1): 155-163. doi:10.1007/s10067-016-3495-3.

\title{
Depressive symptoms and structural disease progression in knee osteoarthritis: data from the Osteoarthritis Initiative
}

\author{
Alan M. Rathbun ${ }^{1}$, Michelle S. Yau ${ }^{2}$, Michelle Shardell ${ }^{3}$, Elizabeth A. Stuart ${ }^{4}$, and Marc C. \\ Hochberg ${ }^{5}$ \\ ${ }^{1}$ Department of Epidemiology and Public Health, University of Maryland School of Medicine, 660 \\ W. Redwood Street, Howard Hall Suite 200, Baltimore, MD 21201, USA \\ ${ }^{2}$ Harvard Medical School, Hebrew SeniorLife Institute for Aging Research, Boston, MA, USA \\ ${ }^{3}$ Translational Gerontology Branch, National Institutes on Aging, Bethesda, MD, USA \\ ${ }^{4}$ Johns Hopkins University Bloomberg School of Public Health, Baltimore, MD, USA \\ ${ }^{5}$ Division of Rheumatology and Clinical Immunology, Department of Medicine, University of \\ Maryland School of Medicine, Baltimore, MD, USA
}

\begin{abstract}
Depressive symptoms are associated with increases in pain and functional limitations in knee osteoarthritis (OA). The aim was to determine whether depressive symptoms are also associated with greater structural knee OA progression. Four years of annual radiographic and clinical assessments from the Osteoarthritis Initiative were analyzed. The Center for Epidemiological Studies Depression Scale was used to identify depressive symptoms (threshold $=\geq 16$ ) at the baseline visit. Propensity scores were used to match participants with and without baseline depressive symptoms on multiple potential confounders. Assessment of radiographic knee OA was based on changes in individual radiographic features, which included osteophyte (OST) grade and joint space narrowing (JSN) grade. Mixed effect models were used to examine structural progression between depressed and non-depressed participants with definitive radiographic knee OA. Depressive symptoms were significantly associated with a higher risk of OST progression (odds ratio $[\mathrm{OR}]=1.74 ; 95 \%$ confidence interval $[\mathrm{CI}]: 1.01,3.00$ ) and a non-significant lower risk of JSN progression ( $\mathrm{OR}=0.40 ; 95 \% \mathrm{CI}: 0.14,1.15) 1$ year after baseline. Conversely, there was a non-significant lower risk of OST progression $(\mathrm{OR}=0.71 ; 95 \% \mathrm{CI}: 0.28,1.79)$ and higher risk of JSN progression (OR $=1.89 ; 95 \%$ CI: $0.71,5.06)$ from year 3 to year 4 of follow-up. However, the patterns of OST progression and JSN progression were not significantly different between the depressed and non-depressed ( $P=0.25$ and 0.15 , respectively). The findings provide no evidence that depressive symptoms have a detectable effect on changes in radiographic disease severity in knee OA.
\end{abstract}

Correspondence to: Alan M. Rathbun.

Compliance with ethical standards

Disclosures None.

Electronic supplementary material The online version of this article (doi:10.1007/s10067-016-3495-3) contains supplementary material, which is available to authorized users. 


\section{Keywords}

Bone; Cartilage; Epidemiology; Osteoarthritis

\section{Introduction}

Depressive symptoms are a frequent comorbidity of osteoarthritis (OA), the most common form of arthritis, which affects approximately 27 million Americans [1, 2]. Meta-analysis estimates indicate the point prevalence of depressive symptoms to be $20 \%$ among those suffering from OA, which is more than twice the prevalence in the general population $[2,3]$. Moreover, depression in OA patients is associated with greater healthcare costs and mortality and decreased quality of life [4, 5]. The medical management of OA patients suffering from co-occurring depressive symptoms is further complicated because this comorbidity often leads to a decrease in intervention efficacy for both the treatment of OA and its sequelae $[6,7]$. Depressive symptoms are often under-recognized by treating rheumatologists, and thus may be under-treated in individuals with arthritis [8].

OA is characterized by structural changes in the subchondral bone and degradation of the joint cartilage, and this pathology is not always accompanied by pain and functional limitations [9]. There is immense heterogeneity in OA patients with respect to the manifestation of OA symptoms, and for example, systematic review evidence suggests that the proportion of patients with radiographic knee OA who report pain ranges widely from 15 to $81 \%$ [10]. Other modifiable clinical factors, such as depressive symptoms, may contribute to the severity and persistency of OA symptoms [11]. Longitudinal studies have consistently demonstrated that depressive symptoms are predictive of changes in pain and functional limitations in OA [12-16]. If depressive symptoms exacerbate OA disease severity, they may also lead to greater structural disease progression.

Depressive symptoms may affect structural disease progression in arthritis patients through biological, psychological, and behavioral mechanisms or by some combination of these processes [17]. Depressive symptoms prime biologically mediated responses to stress and chronic diseases and could exacerbate the degradation of joint cartilage in OA patients through the activation of inflammatory cytokines $[18,19]$. Depressive symptoms are also associated with decreased physical performance, and prolonged periods of sedentary behavior that result in a deconditioning of the body and greater body mass index may lead to greater structural OA disease progression [20, 21]. Existing research has primarily focused on the effect of depressive symptoms on OA symptoms and not explored whether depressive symptoms also affect structural disease progression. Thus, the objective of the current study was to evaluate whether depressive symptoms temporally affect structural disease progression in knee OA. It was hypothesized that the presence of depressive symptoms would be associated with a higher risk of structural disease decline. 


\section{Methods and materials}

\section{Osteoarthritis Initiative study}

Participants for this study came from the Osteoarthritis Initiative (OAI) study, a longitudinal study of knee OA with publicly available data (https://oai.epi-ucsf.org/datarelease/). The OAI is a publicly and privately funded multi-center, prospective, observational cohort study focused on identifying risk factors and biomarkers for development and progression of knee OA [22]. Participants who were at high risk for developing or have developed symptomatic radiographic knee OA were enrolled at four clinical sites within the USA between February 2004 and May 2006: Memorial Hospital of Rhode Island, Ohio State University, the University of Maryland/Johns Hopkins University, and the University of Pittsburgh. Recruitment yielded a racially diverse sample of 4796 men and women between the ages of 45-79 years [22]. All data were collected as part of annual visits and submitted to the OAI data coordinating center at the University of California, San Francisco. Institutional review boards at each OAI clinical site and the OAI coordinating center (University of California, San Francisco) approved the OAI study. The publically available OAI data are de-identified, and participant information cannot be extrapolated back to any particular individual.

Approval for the current study was not required in accordance with the policies of the participating institutions.

\section{Study sample}

The analytic sample was drawn from participants with established radiographic disease ( $N=$ 2432) that was defined using a Kellgren-Lawrence (K-L) grade of two or three in one or both knees at study enrollment (Fig. 1). Subjects with end-stage OA disease in one or both knees $(n=292)$, defined by a K-L grade of four or evidence of total knee replacement at baseline, were excluded. For participants with radiographic evidence of OA in both knees, only the knee from the dominant leg was included in the analysis. The dominant leg was assigned using the following algorithm: (1) leg used to kick a ball, (2) dominant hand if ambidextrous legs or missing dominant leg data, and (3) right leg if missing data on dominant leg or hand. The sample was further restricted to participants $(n=2255)$ with complete baseline data on variables used in analyses (detailed below). Subjects lacking a follow-up visit $(n=5)$ with radiographic data were also excluded, as well as those with a knee that met the maximum medial compartment OST grade $(n=65)$ or JSN grade $(n=0)$.

\section{Depressive symptoms}

The long form 20-Item Center for Epidemiological Studies Depression (CES-D) Scale was used to assess depressive symptoms at study enrollment [23]. Validation research has shown the CES-D to be a valid and reliable measure of depressive symptoms among older adults with multiple chronic conditions [24]. The recommended screening threshold of $\geq 16$ to identify depressive symptoms has been demonstrated to have good screening properties when validated against clinically diagnosed major depressive disorder and was used to classify the presence of depressive symptoms upon entry into the OAI cohort [25]. To assess the potential effects of bias resulting from exposure misclassification, sensitivity analyses were performed that replicated the primary study using a stricter CES-D cut point (threshold $=\geq 19)$ to classify the presence of depressive symptoms at study baseline [25]. 


\section{Definition of radiographic progression}

Bilateral posteroanterior weight-bearing fixed-flexion knee radiographs were obtained by trained and certified radiologic technicians using a Synaflexer ${ }^{\mathrm{TM}}$ platform (Synarc, San Francisco, CA) and were read centrally at Boston University [26]. In order to precisely determine how depressive symptoms affect the structural pathology of OA, the progression outcome variables were based on individual radiographic features: osteophyte (OST) grade (range 0-6) and joint space narrowing (JSN) grade (range 0-3). OST grade was assessed as the sum of the tibial and femoral grades for the medial side to obtain a cumulative sum for the medial compartment. Medial compartment JSN grade was rounded to the nearest whole number (e.g., $1.8 \rightarrow 2.0$ ). OST and JSN progression (yes or no) at each follow-up visit were defined as whether the grade of OST and JSN increased (e.g., $1 \rightarrow 2$ ) from the previous visit. Subjects' structural disease progression was evaluated moving forward through time from baseline at follow-up visits with available radiographic X-ray data as they continually progressed between time points (Fig. 1). Four-year follow-up data were analyzed because it was the time hypothesized necessary to observe changes in structural disease progression that may be related to the presence of depressive symptoms at study baseline. Participants were right censored when they reached the maximum grade for the outcome of interest.

\section{Potential confounders}

Potential confounders measured at study baseline were selected a priori based on literature review. Demographic, socioeconomic, and behavioral measures included age (years), sex, race (white, African American, Asian, or other), marital status (married, widowed, divorced, separated, or never married), education (high school graduate, college graduate, or graduate degree), health insurance (insured or uninsured), employment (employed or unemployed), smoking (never, former, or former), alcohol consumption (none, minimal, or moderate), and physical activity. Physical activity was measured using the Physical Activity Scale for the Elderly [27]. Clinical characteristics were body mass index (BMI), comorbidity, symptomatic OA, history of knee injury, analgesic use, pain, functional disability, and joint stiffness. Comorbidity was assessed using the Charlson Comorbidity Index, which does not incorporate major depression [28]. Symptomatic OA was defined as "pain, aching or stiffness in or around the knee on most days" for at least 1 month during the past 12 months. Knee injury was operationalized as "ever injured badly enough to limit ability to walk for at least two days." Analgesic use included acetaminophen and non-steroidal anti-inflammatory drugs including COX-2 selective inhibitors. OA symptoms were assessed using the summary score of the Western Ontario and McMaster Universities Osteoarthritis (WOMAC) Index [29]. Structural disease severity was assessed using K-L grade [30].

\section{Matching}

Propensity score (PS) matching was used to account for potential confounding factors, particularly baseline differences in OA disease severity, and other factors that can influence structural progression. Arthritis disease severity exhibits strong intra-person clustering, and baseline values are the strongest predictor of subsequent values. Without accounting for baseline OA disease severity, any relationship between depressive symptoms and structural progression could be due to residual confounding. Specifically, depressed patients could 
progress more rapidly because they have worse baseline disease severity, rather than depressive symptoms affecting changes in radiographic disease through a substantive impact on structural disease progression. Total WOMAC score and $\mathrm{K}-\mathrm{L}$ grade at study enrollment were considered possible confounders.

PS matching was used for two reasons. First, OA disease severity measures are correlated and collinearity cannot directly bias effect estimates. Second, PS matching permits direct comparisons without complex longitudinal modeling adjusting for the main effect of baseline covariates and their interaction with follow-up time. Subjects with baseline depressive symptoms were matched to controls with no baseline symptoms of depression. The propensity score model incorporated all baseline variables selected a priori as potential confounders: age, sex, race, marital status, education, employment status, health insurance, smoking, alcohol consumption, physical activity, BMI, comorbidity, symptomatic OA, analgesic use, history of knee injury, $\mathrm{K}-\mathrm{L}$ grade, and total WOMAC score. Logistic regression modeling was used to calculate a propensity score for each subject: the estimated probability of baseline depressive symptoms conditional on potential confounders. Depressed subjects were matched without replacement to non-depressed controls at a ratio of 1 to 2 using nearest neighbor matching and a caliper of 0.05 .

\section{Statistical analysis}

Participant characteristics were compared between patients with and without baseline depressive symptoms. Means and standard deviations were estimated for continuous variables, and $t$ tests and standardized mean differences were used to assess for differences between the groups. For categorical measures, percentages were calculated and Chi-square tests and standardized mean differences were used to examine differences between the groups. Descriptive analyses were performed separately in the original analytic sample and the matched sample to assess the balancing of covariates after matching.

Mixed effect models were used to evaluate differences in changes in structural disease progression between participants with and without baseline depressive symptoms. Outcomes included radiographic measures of OA disease severity (OST grade and JSN grade) that were modeled as a time-varying binary indictor variable for progression to a higher grade (i.e., disease progression) at each follow-up visit. Locally weighted scatterplot smoothing curves were used to examine longitudinal functional forms (probability of structural disease progression over time) from years 1 through 4 (probability of structural disease progression at baseline is zero). Time was modeled using categorical indicators of years since baseline to allow for non-linear functional forms. The design of OAI is hierarchical; observations are nested within subjects, which are clustered within-clinical sites. A random intercept was included to address the correlation of observations within-subjects; random effects (intercept) for within-site correlation and for within-matched pair correlation did not improve model fit. 


\section{Results}

\section{Propensity score matching}

Approximately $10.7 \%(n=212)$ of the original analytic sample $(n=1973)$ had prevalent depressive symptoms. Depressed participants were more likely to be younger, female, nonwhite, not married, smokers, non-drinkers, and of lower socioeconomic status as measured by educational attainment and health insurance (Table 1). Depressive symptoms were also associated with lower $\mathrm{K}-\mathrm{L}$ grade and greater BMI, co-morbidity, symptomatic OA, analgesic use, and total WOMAC score. Depressed subjects were propensity score matched $(1: 2)$ to non-depressed controls, and $78.3 \%(n=166)$ of those with prevalent depressive symptoms were retained. Excluded subjects who could not be matched had insufficient overlap in propensity score distributions (Fig. S1). Matching resulted in a rebalancing of characteristics that were associated with prevalent depressive symptoms at study baseline (Fig. S2).

\section{OST progression}

In the matched sample, the risk of moving to a higher OST grade was significantly greater in the depressed than the non-depressed 1 year after baseline: $30.7 \%$ (46/150) vs $22.0 \%$ (67/304) (Table 2$)$, respectively $(P=0.04)$. Conversely, from year 3 to year 4 , the depressed had a non-significantly lower likelihood of OST progression compared to the non-depressed: $7.1 \%(8 / 112)$ vs $9.6 \%(23 / 240)$, respectively $(P=0.46)$. The 1 -year probability decreased faster in the depressed than in the non-depressed (Fig. 2), evidenced by the decreasing OR for OST progression associated with depressive symptoms: OR $=1.74$ (95\% confidence interval $[\mathrm{CI}]: 1.01,3.00)$ from baseline to year $1 ; \mathrm{OR}=1.37$ (95\% CI: $0.68,2.77)$ from year 1 to year 2 ; OR $=1.07$ (95\% CI: $0.51,2.24)$ from year 2 to year 3 ; and $\mathrm{OR}=0.71(95 \% \mathrm{CI}$ : $0.28,1.79$ ) from year 3 to year 4 (Table 2 ). However, the global test of depressive symptoms and depressive symptom by follow-up time interaction was not statistically significant $(P=$ $0.25)$.

The results for OST progression between depressed and non-depressed subjects were similar when defining depressive symptoms using a stricter CES-D threshold (data not shown).

\section{JSN progression}

Among the matched participants, the proportion of depressed subjects moving to a higher JSN grade increased from $3.3 \%(5 / 150) 1$ year after baseline to $8.0 \%(9 / 112)$ from year 3 to year 4 (Table 2), and this within-group change approached statistical significance $(P=0.07)$. In contrast, the percentage of non-depressed participants experiencing JSN progression decreased from 7.6\% (23/304) 1 year after baseline to $4.7 \%$ (11/236) from year 3 to year 4 , respectively $(P=0.25)$. Thus, the risk for JSN progression was increasing in the depressed and decreasing in the non-depressed (Fig. 3). The 1-year associations comparing the risk of JSN progression between the depressed and non-depressed were as follows: $\mathrm{OR}=0.40$ (95\% CI: $0.14,1.15)$ from baseline to year $1 ; 0.47$ (95\% CI: $0.15,1.50)$ from year 1 to year 2; 0.69 (95\% CI: 0.24, 1.93) from year 2 to year 3; and 1.89 (95\% CI: 0.71, 5.06) from year 3 to year 4 (Table 2). However, neither the time-specific differences nor the global test of depressive symptoms and depressive symptom by follow-up time interaction were 
statistically significant $(P=0.15)$. Similarly, findings from sensitivity analyses using a more stringent CES-D cut point did not alter the interpretation of the observed results (data not shown).

\section{Discussion}

This is the first study specifically designed to examine whether depressive symptoms have an effect on the time-varying probability of structural disease progression in knee OA. The risk of OST progression 1 year after baseline was significantly higher in depressed than in non-depressed participants: a discrepancy that diminished at later time points. By contrast, participants with symptoms of depression had a non-significant lower risk for JSN progression from baseline to 1 year, but the 1-year probability of progression increased in depressed and decreased in non-depressed participants, and those with depressive symptoms had a non-significant higher risk for JSN progression from year 3 to year 4. Given that the differences in the trajectories of structural disease progression between depressed and nondepressed subjects were not statistically significant, the impact of depressive symptoms on structural progression defined using measures of radiographic disease severity may be minimal and too small to detect.

The temporal effect of depressive symptoms on OST progression compared to JSN progression manifested differently over time. Osteophytes are bony outgrowths occurring at the joint margins associated with the loss of cartilage, and research suggests that they are a marker of joint healing that develop during the bone remodeling process in response to excessive joint loading from weight, malalignment, or other biomechanical features [31]. JSN is indicative of degeneration in cartilage tissue and has been used as the primary definition for structural disease progression in OA [30]. If depressive symptoms cause greater pain and functional limitations through their impact on inflammatory mediators and/or joint loading, then increases in OST progression could be the proximal structural disease outcome and, ultimately, greater cartilage loss and JSN [19, 21]. Riddle and colleagues demonstrated that psychological distress was significantly associated with a greater risk of rapid progression to knee arthroplasty [32]. However, findings from the current research and one previous study indicate that the increased risk of radiographic progression (e.g., JSN) associated with depressive symptoms is not statistically significant [33]. As OA symptoms are predictive of OST progression and JSN progression, the nonsignificant associations between depressive symptoms and radiographic disease severity may be representative of more severe OA phenotypes [34].

Depressive symptoms in knee OA are likely a consequence of greater disease severity, and these participants could also inherently be at a higher risk for subsequent structural progression because of poorer prognostic factors, in addition to depressed mood [34, 35]. OA may have unique phenotypes, comprising distinct subgroups with different constellations of characteristics, and clusters with certain predisposing risk factors may have faster rates of structural disease progression compared to other subgroups [34, 36]. Clinically relevant predictors of structural disease progression include age, body composition, acute joint injury, functional limitations, and pain, which were highly correlated with baseline depressive symptoms in the original analytic sample, as evidenced 
by the standardized mean differences that were as large as 0.75 standard deviations [34, 37, 38]. These factors may collectively contribute to the excess risk for depressed mood in OA patients, but the diametrically opposed influence and distal impact of depressive symptoms may not be large enough in magnitude to result in a detectable effect on structural disease progression [15], although it is also possible that the effects of depressive symptoms on structural disease progression may be perceptible when using more sensitive methods (e.g., magnetic resonance imaging $[\mathrm{MRI}]$ ) to evaluate structural disease changes. Moreover, patient-reported OA symptoms form the basis for clinical decision making regarding surgical intervention, and depressive symptoms could create an increased risk of knee arthroplasty, independent of any impact on radiographic disease [32].

There are limitations to this research. Foremost, radiographic OA and depressive symptoms were defined at the same time point, which has the potential to cause collider bias via selection effects and, in part, could potentially contribute to the null findings [39]. However, such confounding is generally small and would need to be very large in magnitude to have a substantive impact on the results [39]. Matching and the small overall outcome frequencies may have further reduced power and the ability to detect differences in structural progression. Depressive symptoms were measured using a self-reported questionnaire, but participants with case-level mood disorder have more severe depressive symptoms, and the effects in these individuals could be large enough to detect. Depression is also dynamic, and prior depressive illness predicts future depressive symptoms that manifest with greater frequency and intensity, but the current study design using a time-invariant exposure definition may underestimate the observed associations [40]. Lastly, there is the potential for confounding by unmeasured factors not included in the analysis.

The weaknesses of this study are mollified through the various strengths. A multi-site, prospective, observational cohort was used that provided a large heterogeneous sample of participants with radiographic knee OA [22]. The OAI was specifically designed to investigate determinants of disease progression and contains comprehensive and validated measures of radiographic and symptomatic $\mathrm{OA}$ and salient clinical characteristics that permitted robust adjustment for a range of potential confounders [22]. The findings were also robust to exposure misclassification that could arise from depression measurement using a patient-reported survey and suggest that the observed associations are consistent across different levels of depressive symptom severity. Structural progression was operationalized using a time-varying definition, and yearly association measures were reported to allow for clear interpretations regarding the risks of structural decline associated with baseline depressive symptoms. This study is the first specifically designed using robust statistical methods to prospectively evaluate depressive symptoms and their relationship to the course of structural disease progression in knee OA.

In summary, the results provide important clinical information, indicating that the presence of depressive symptoms in knee OA may not significantly affect changes in radiographic disease severity. The statistically and clinically significant effects of depressive symptoms on OA disease severity may be limited to patients' experience of their OA symptoms and choice for surgical intervention, i.e., pain, functional limitations, and knee arthroplasty. Rather than depressed mood being a uniquely distinct clinical OA phenotype, depressive 
symptoms may simply be another constituent among a larger set of prognostic factors in OA subgroups that have faster structural progression and more severe OA disease severity. Thus, the clinical management of this comorbidity should be considered as another component in the broader context of the different factors that contribute to clinical OA care. Future studies should determine whether depressive symptoms affect structural disease changes as assessed using MRI and if they impact the choice for surgical intervention in knee OA.

\section{Supplementary Material}

Refer to Web version on PubMed Central for supplementary material.

\section{Acknowledgments}

The OAI is a public-private partnership composed of five contracts (N01-AR-2-2258; N01-AR-2-2259; N01AR-2-2260; N01-AR-2-2261; N01-AR-2-2262) funded by the National Institutes of Health, a branch of the Department of Health and Human Services, and conducted by the OAI Study Investigators. Private funding partners include Merck Research Laboratories; Novartis Pharmaceuticals Corporation, GlaxoSmithKline; and Pfizer, Inc. Private sector funding for the OAI is managed by the Foundation for the National Institutes of Health. This manuscript was prepared using an OAI public use data set and does not necessarily reflect the opinions or views of the OAI investigators, the $\mathrm{NIH}$, or the private funding partners.

Funding This research was supported by a training grant (T32 AG000262) from the National Institute on Aging.

\section{References}

1. Lawrence RC, Felson DT, Helmick CG, Arnold LM, Choi H, Deyo RA, et al. Estimates of the prevalence of arthritis and other rheumatic conditions in the United States: part II. Arthritis Rheum. 2008; 58(1):26-35. [PubMed: 18163497]

2. Stubbs B, Aluko Y, Myint PK, Smith TO. Prevalence of depressive symptoms and anxiety in osteoarthritis: a systematic review and meta-analysis. Age Ageing. 2016; 45(2):228-235. [PubMed: 26795974]

3. Kessler RC, Berglund P, Demler O, Jin R, Koretz D, Merikangas KR, et al. The epidemiology of major depressive disorder: results from the National Comorbidity Survey Replication (NCS-R). JAMA. 2003; 289(23):3095-3105. [PubMed: 12813115]

4. Rosemann T, Gensichen J, Sauer N, Laux G, Szecsenyi J. The impact of concomitant depression on quality of life and health service utilisation in patients with osteoarthritis. Rheumatol Int. 2007; 27(9):859-863. [PubMed: 17242902]

5. Lee TA, Shields AE, Vogeli C, Gibson TB, Woong-Sohn M, Marder WD, et al. Mortality rate in veterans with multiple chronic conditions. J Gen Intern Med. 2007; 22(3):403-407. [PubMed: 18026809]

6. CG177 N. Osteoarthritis Care and Management in Adults. London: National Institute for Health and Care Excellence; 2014.

7. Detweiler-Bedell JB, Friedman MA, Leventhal H, Miller IW, Leventhal EA. Integrating co-morbid depression and chronic physical disease management: identifying and resolving failures in selfregulation. Clin Psychol Rev. 2008; 28(8):1426-1446. [PubMed: 18848740]

8. Rathbun AM, Harrold LR, Reed GW. A description of patient- and rheumatologist-reported depression symptoms in an American rheumatoid arthritis registry population. Clin Exp Rheumatol. 2014; 32(4):523-532. [PubMed: 24984165]

9. Lane N, Brandt K, Hawker G, Peeva E, Schreyer E, Tsuji W, et al. OARSI-FDA initiative: defining the disease state of osteoarthritis. Osteoarthr Cartil. 2011; 19(5):478-482. [PubMed: 21396464]

10. Bedson J, Croft PR. The discordance between clinical and radiographic knee osteoarthritis: a systematic search and summary of the literature. BMC Musculoskelet Disord. 2008; 9:116. [PubMed: 18764949] 
11. Cohen E, Lee YC. A mechanism-based approach to the management of osteoarthritis pain. Curr Osteoporos Reports. 2015; 13(6):399-406.

12. Zautra AJ, Smith BW. Depression and reactivity to stress in older women with rheumatoid arthritis and osteoarthritis. Psychosom Med. 2001; 63(4):687-696. [PubMed: 11485123]

13. Dunlop DD, Semanik P, Song J, Manheim LM, Shih V, Chang RW. Risk factors for functional decline in older adults with arthritis. Arthritis Rheum. 2005; 52(4):1274-1282. [PubMed: 15818691]

14. Kroenke K, Wu J, Bair MJ, Krebs EE, Damush TM, Tu W. Reciprocal relationship between pain and depression: a 12-month longitudinal analysis in primary care. J Pain. 2011; 12(9):964-973. [PubMed: 21680251]

15. Riddle DL, Kong X, Fitzgerald GK. Psychological health impact on 2-year changes in pain and function in persons with knee pain: data from the osteoarthritis initiative. Osteoarthr Cartil. 2011; 19(9):1095-1101. [PubMed: 21723400]

16. Collins J, Katz J, Dervan E, Losina E. Trajectories and risk profiles of pain in persons with radiographic, symptomatic knee osteoarthritis: data from the osteoarthritis initiative. Osteoarthr Cartil. 2014; 22(5):622-630. [PubMed: 24662734]

17. Rathbun AM, Reed GW, Harrold LR. The temporal relationship between depression and rheumatoid arthritis disease activity, treatment persistence and response: a systematic review. Rheumatology (Oxford). 2013; 52(10):1785-1794. [PubMed: 23236191]

18. Rohleder N. Stimulation of systemic low-grade inflammation by psychosocial stress. Psychosom Med. 2014; 76(3):181-189. [PubMed: 24608036]

19. Rahmati M, Mobasheri A, Mozafari M. Inflammatory mediators in osteoarthritis: a critical review of the state-of-the-art, current prospects, and future challenges. Bone. 2016; 85:81-90. [PubMed: 26812612]

20. Demakakos P, Cooper R, Hamer M, de Oliveira C, Hardy R, Breeze E. The bidirectional association between depressive symptoms and gait speed: evidence from the English longitudinal study of ageing (ELSA). PLoS One. 2013; 8(7):e68632. [PubMed: 23874698]

21. Beckwee D, Vaes P, Shahabpour M, Muyldermans R, Rommers N, Bautmans I. The influence of joint loading on bone marrow lesions in the knee: a systematic review with meta-analysis. Am $\mathrm{J}$ Sports Med. 2015; 43(12):3093-3107. [PubMed: 25634907]

22. Nevitt, M., Felson, D., Lester, G. The osteoarthritis initiative. University of California; San Francisco: 2006.

23. Radloff LS. The CES-D scale a self-report depression scale for research in the general population. Appl Psychol Meas. 1977; 1(3):385-401.

24. Zauszniewski JA, Bekhet AK. Depressive symptoms in elderly women with chronic conditions: measurement issues. Aging Mental Health. 2009; 13(1):64-72. [PubMed: 19197691]

25. Smarr KL, Keefer AL. Measures of depression and depressive symptoms: Beck depression inventory-II (BDI-II), Center for Epidemiologic Studies Depression Scale (CES-D), geriatric depression scale (GDS), hospital anxiety and depression scale (HADS), and patient health questionnaire-9 (PHQ-9). Arthritis Care Res. 2011; 63(S11):S454-S466.

26. Altman RD, Gold G. Atlas of individual radiographic features in osteoarthritis, revised. Osteoarthr Cartil. 2007; 15:A1-A56. [PubMed: 17320422]

27. Washburn RA, Smith KW, Jette AM, Janney CA. The physical activity scale for the elderly (PASE): development and evaluation. J Clin Epidemiol. 1993; 46(2):153-162. [PubMed: 8437031]

28. Charlson M, Szatrowski TP, Peterson J, Gold J. Validation of a combined comorbidity index. J Clin Epidemiol. 1994; 47(11):1245-1251. [PubMed: 7722560]

29. Bellamy N. Validation study of WOMAC: a health status instrument for measuring clinicallyimportant patient-relevant outcomes following total hip or knee arthroplasty in osteoarthritis. J Orthop Rheumatol. 1988; 1:95-108.

30. KELLGREN JH, LAWRENCE JS. Radiological assessment of osteoarthrosis. Ann Rheum Dis. 1957; 16(4):494-502. [PubMed: 13498604]

31. Felson DT, Gale DR, Elon Gale M, Niu J, Hunter DJ, Goggins J, et al. Osteophytes and progression of knee osteoarthritis. Rheumatology (Oxford). 2005; 44(1):100-10. 4. [PubMed: 15381791] 
32. Riddle DL, Kong X, Jiranek WA. Factors associated with rapid progression to knee arthroplasty: complete analysis of three-year data from the osteoarthritis initiative. Joint Bone Spine. 2012; 79(3):298-303. [PubMed: 21727020]

33. Wolfe F, Lane NE. The longterm outcome of osteoarthritis: rates and predictors of joint space narrowing in symptomatic patients with knee osteoarthritis. J Rheumatol. 2002; 29(1):139-146. [PubMed: 11824950]

34. Karsdal M, Bihlet A, Byrjalsen I, Alexandersen P, Ladel C, Michaels M, et al. OA phenotypes, rather than disease stage, drive structural progression-identification of structural progressors from 2 phase III randomized clinical studies with symptomatic knee OA. Osteoarthr Cartil. 2015; 23(4): 550-558. [PubMed: 25576879]

35. Hawker GA, Gignac MA, Badley E, Davis AM, French MR, Li Y, et al. A longitudinal study to explain the pain-depression link in older adults with osteoarthritis. Arthritis Care Res. 2011; 63(10):1382-1390.

36. Van der Esch M, Knoop J, van der Leeden M, Roorda L, Lems W, Knol D, et al. Clinical phenotypes in patients with knee osteoarthritis: a study in the Amsterdam osteoarthritis cohort. Osteoarthr Cartil. 2015; 23(4):544-549. [PubMed: 25596322]

37. Bastick AN, Belo JN, Runhaar J, Bierma-Zeinstra SM. What are the prognostic factors for radiographic progression of knee osteoarthritis? A meta-analysis. Clin Orthop Relat Res. 2015; 473(9):2969-2989. [PubMed: 25995176]

38. Cooper C, Snow S, McAlindon TE, Kellingray S, Stuart B, Coggon D, et al. Risk factors for the incidence and progression of radiographic knee osteoarthritis. Arthritis Rheum. 2000; 43(5):995. [PubMed: 10817551]

39. Zhang Y, Niu J, Felson DT, Choi HK, Nevitt M, Neogi T. Methodologic challenges in studying risk factors for progression of knee osteoarthritis. Arthritis Care Res. 2010; 62(11):1527-1532.

40. Monroe SM, Slavich GM, Gotlib IH. Life stress and family history for depression: the moderating role of past depressive episodes. J Psychiatr Res. 2014; 49:90-95. [PubMed: 24308926] 


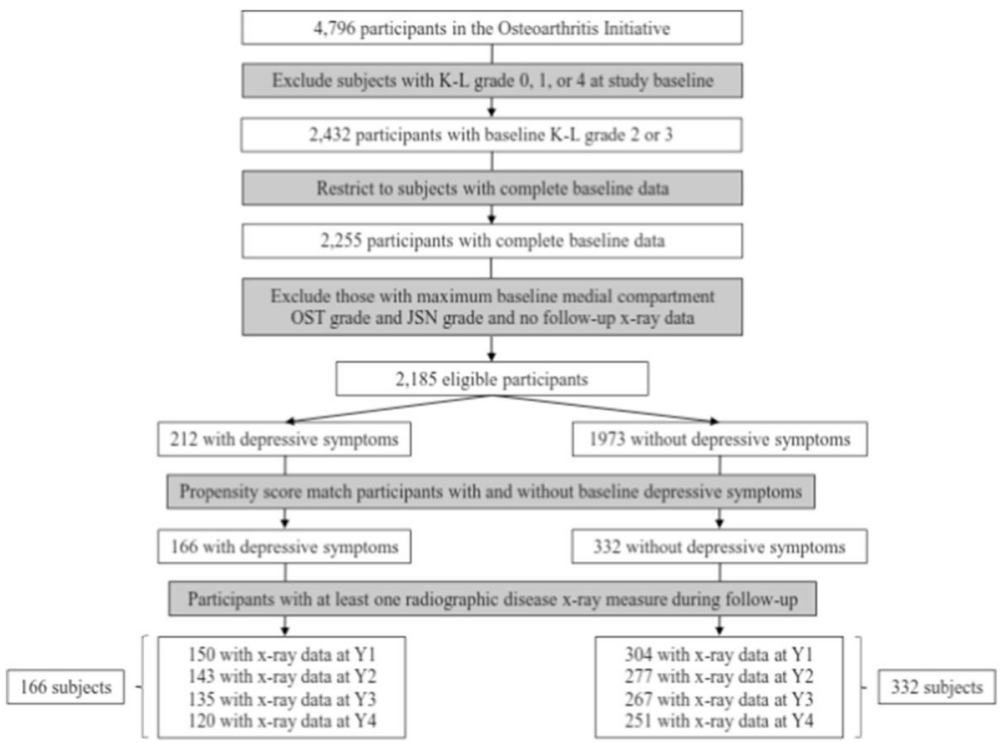

Fig. 1.

Study sample flow diagram. $K-L$ Kellgren-Lawrence, $J S N$ joint space narrowing, $O S T$ osteophyte, $Y 1$ year 1, Y2 year 2, Y3 year 3, Y4 year 4 


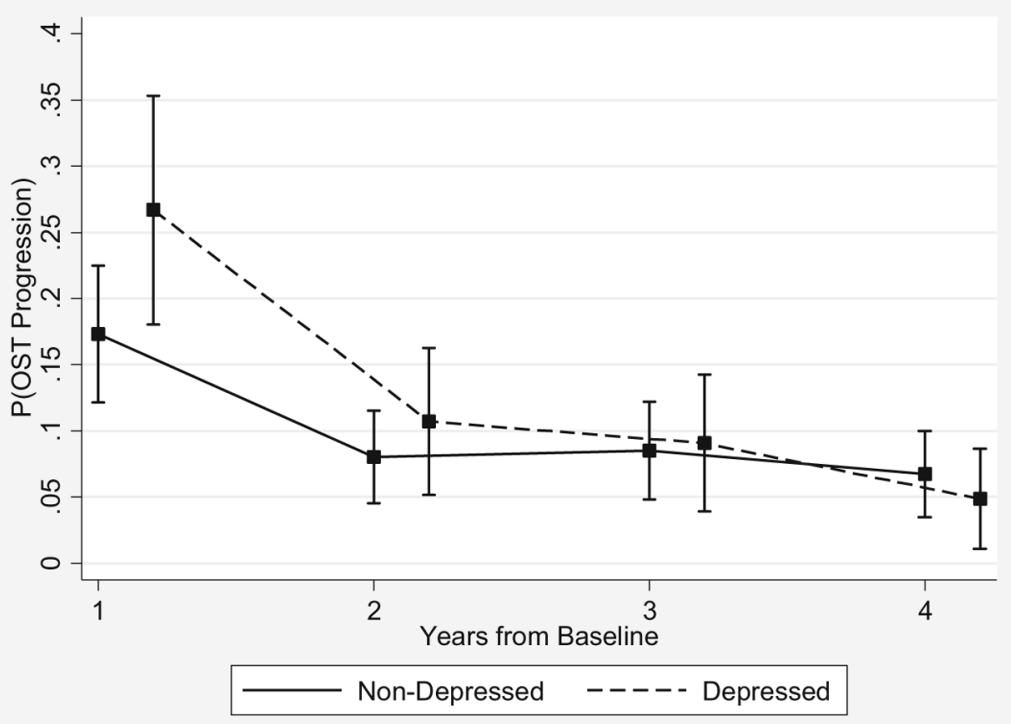

Fig. 2.

Probability of OST progression during follow-up in the OAI comparing depressed and nondepressed propensity score matched subjects. OAIOsteoarthritis Initiative, OST osteophyte 


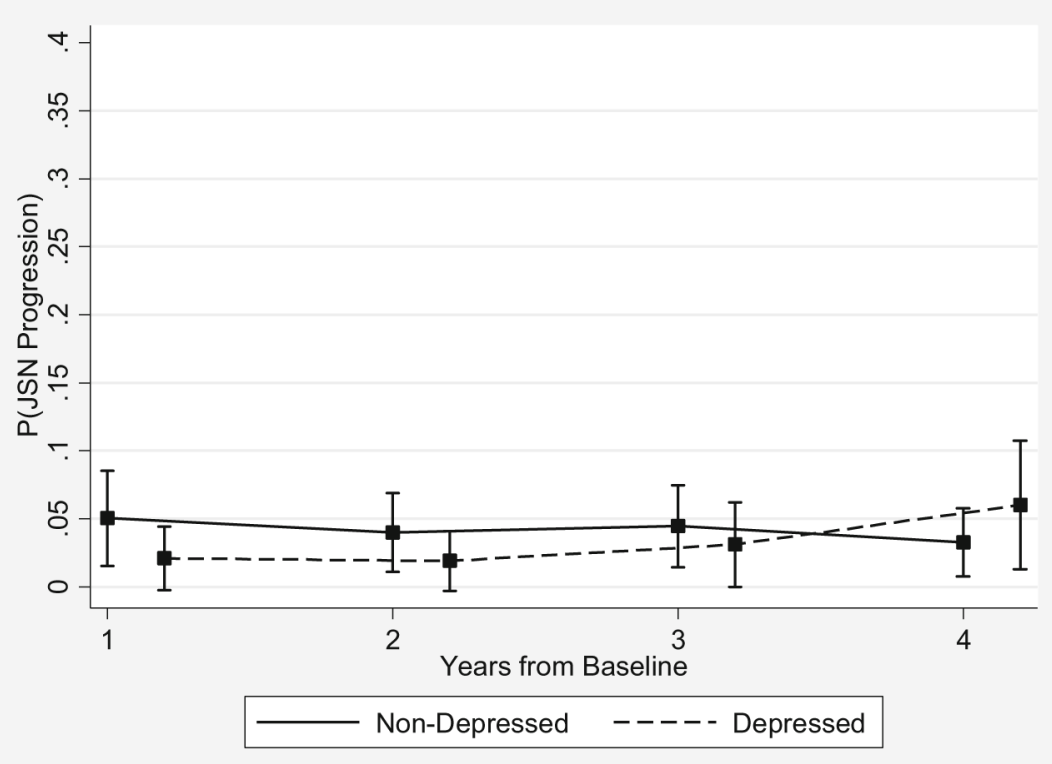

Fig. 3.

Probability of JSN progression during follow-up in the OAI comparing depressed and nondepressed propensity score matched subjects. JSN joint space narrowing, OAIOsteoarthritis Initiative 
Rathbun et al.

Page 16

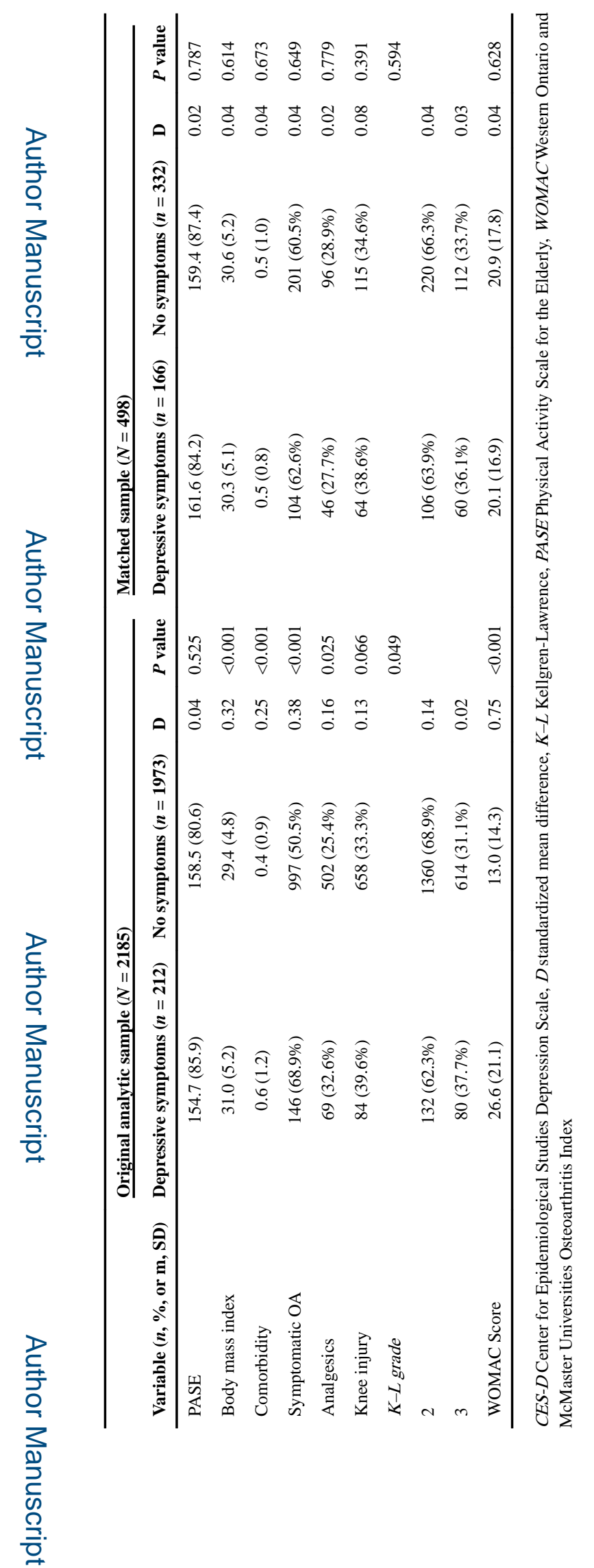

Clin Rheumatol. Author manuscript; available in PMC 2018 January 01. 


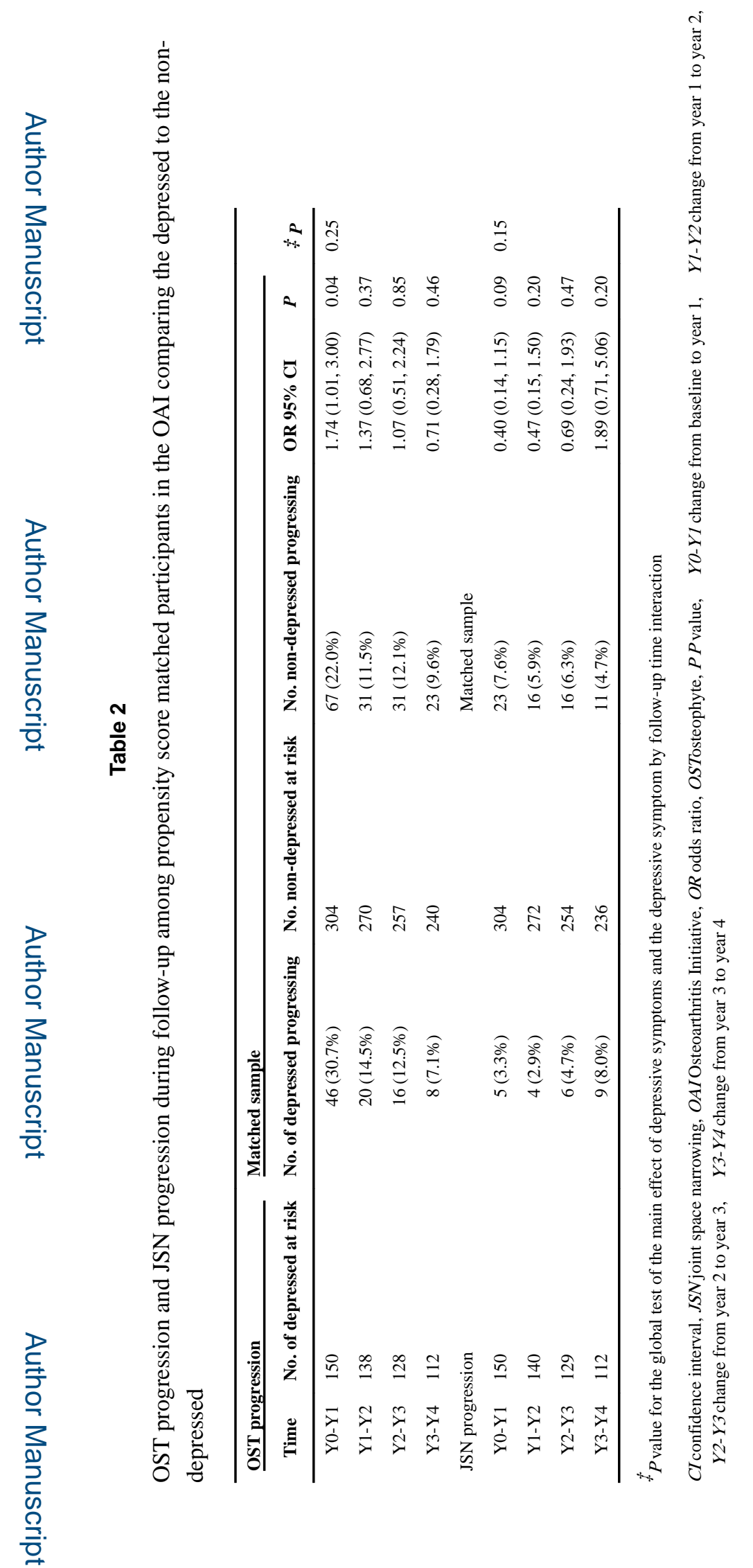

Clin Rheumatol. Author manuscript; available in PMC 2018 January 01. 